and so lose energy: interactions with ordinary matter and light can brake the acceleration at a maximum energy below the limits imposed by time or space.

Shock acceleration at supernova remnants can explain cosmic-ray energies up to about $10^{15} \mathrm{eV}$, where, interestingly, the cosmic-ray spectrum suddenly turns downwards (Fig. 1). But we still observe some cosmic rays to energies six orders of magnitude above this 'knee'. Perhaps the higher-energy cosmic rays come from an entirely different source, so the spectrum we see is the sum of two separate spectra (such as the two bottom curves in Fig. 1). Alternatively, acceleration of lower-energy cosmic rays may continue to higher energies, either at shock waves in magnetic stellar winds, or by continuous acceleration in the interstellar medium (by random scatterings from moving magnetic irregularities). In Fig. 1, the central green curve depicts this possibility.

Now the picture may have become more complicated. Erlykin and Wolfendale ${ }^{1}$ point out that much of the cosmic-ray flux at Earth could be from the most recent nearby supernova. For cosmic rays coming from a single source, the time and space scales are fixed, so each type of nucleus should have a sharply defined maximum energy, proportional to its charge. This would produce a sawtooth pattern in the spectrum near the knee, each 'tooth' corresponding to an abundant element (Fig. 1, top).

Cosmic rays at these high energies are detected from the 'air showers' that they produce. A cosmic-ray particle enters the Earth's atmosphere and collides with the nucleus of an atom to produce a large number of secondary particles, which hit other nuclei, and so on. At ground level, this translates to a shower of electrons, positrons, photons and muons, whose size depends on the energy of the original cosmic ray. Collating all available data from air-shower experiments, Erlykin and Wolfendale argue that they have discovered 'teeth' corresponding to oxygen and iron near the knee, signs of a recent nearby supernova. Such a supernova would need to be much younger than $10^{5}$ years, and would have to have occurred much closer than $1 \mathrm{kpc}$. Thus, Vela is a possibility, for instance. If confirmed, this would be powerful evidence for the supernova-remnant theory of cosmic-ray acceleration.

Experimental data now in the pipeline should shed more light on the chemical composition, and thus the origin, of particles with energies from the knee, near $10^{15}$ $\mathrm{eV}$, up to $10^{18} \mathrm{eV}$, forming a vital bridge to the highest-energy scales. At the highest energies, above $10^{18} \mathrm{eV}$, cosmic-ray particles can no longer be contained by our Galaxy's magnetic fields; so as Giuseppe Cocconi realized forty years $\mathrm{ago}^{5}$, these particles must come from beyond our Milky Way. It is the goal of the international Auger project - a pair of detector arrays each covering 3,000 square kilometres, still in the planning stage - to detect these highest-energy cosmic-ray particles. To understand their origin and propagation through the Universe would turn cosmic-ray physics into a tool of fundamental physics at extreme energies.
Peter L. Biermann is in the Max Planck Institute for Radioastronomy, D-53010 Bonn, Germany.

1. Erlykin, A. \& Wolfendale, A. Astroparticle Phys. 7, 1-12 (1997).

2. Baade, W. \& Zwicky, F. Proc. Natl Acad. Sci. USA 20, 259-263 (1934).

3. Fermi, E. Phys. Rev. 75, 1169-1174 (1949).

4. Fermi, E. Astrophys. J. 119, 1-6 (1954)

5. Cocconi, G. Nuovo Cimento 3, 1433-1442 (1956).

\title{
Palaeocinatology
}

\section{Back at the last interglacial}

\section{Joël Guiot}

$\mathrm{t}$ has been established that, over the past 20,000 years or so, alterations in regional vegetation are controlled by the broad, global pattern of climate change. But what about periods longer than this? Are non-climatic factors such as migration and competition between species involved? The answer to this question is of interest because it can tell us how much we can learn about past climate from the vegetation of the time.

On page 57 of this issue ${ }^{1}$, Whitlock and Bartlein describe their analysis of a 125,000year pollen record of vegetation from Carp Lake in the Pacific northwest of North America. Their conclusion is that climate variations are the cause of the millennial-scale changes in vegetation that they see; that is, the fluctuations between steppe and forest vegetation cover, and between subalpine forest and forest types adapted to warm and dry conditions, are strongly correlated with global climate see Fig. 2 of the paper, page 58. So low ice volume with maximum summer insolation favoured 'warm-and-dry' forest, and moderate ice volume with minimum summer insolation supported subalpine conifers.

It is then possible to assign vegetation to particular climatic events. So-called stage $5 \mathrm{e}$ (the Eemian, the last interglacial before the present one, which ran from about 125,000 to 115,000 years ago) was characterized by warm-and-dry, full forest. Stage $5 \mathrm{a}$ (the last temperate period before the ensuing full glacial, 85,000-75,000 years ago) was, by contrast, characterized by a warm-and-dry but less dense forest, composed of both trees

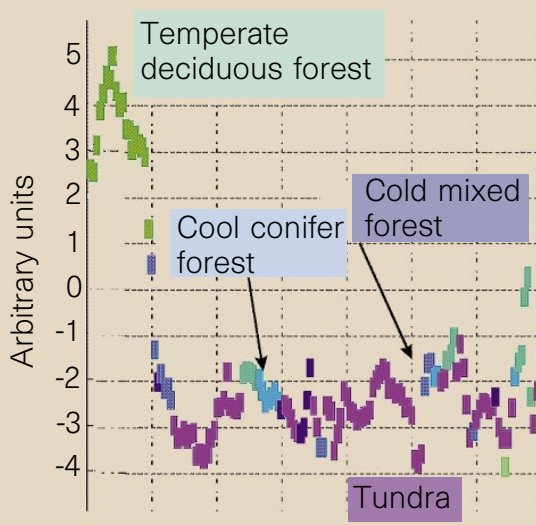

\section{Cool mixed}
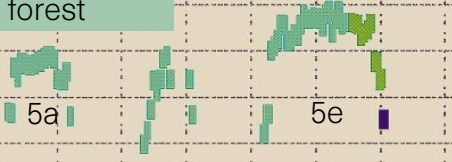

i $\quad i \quad i$
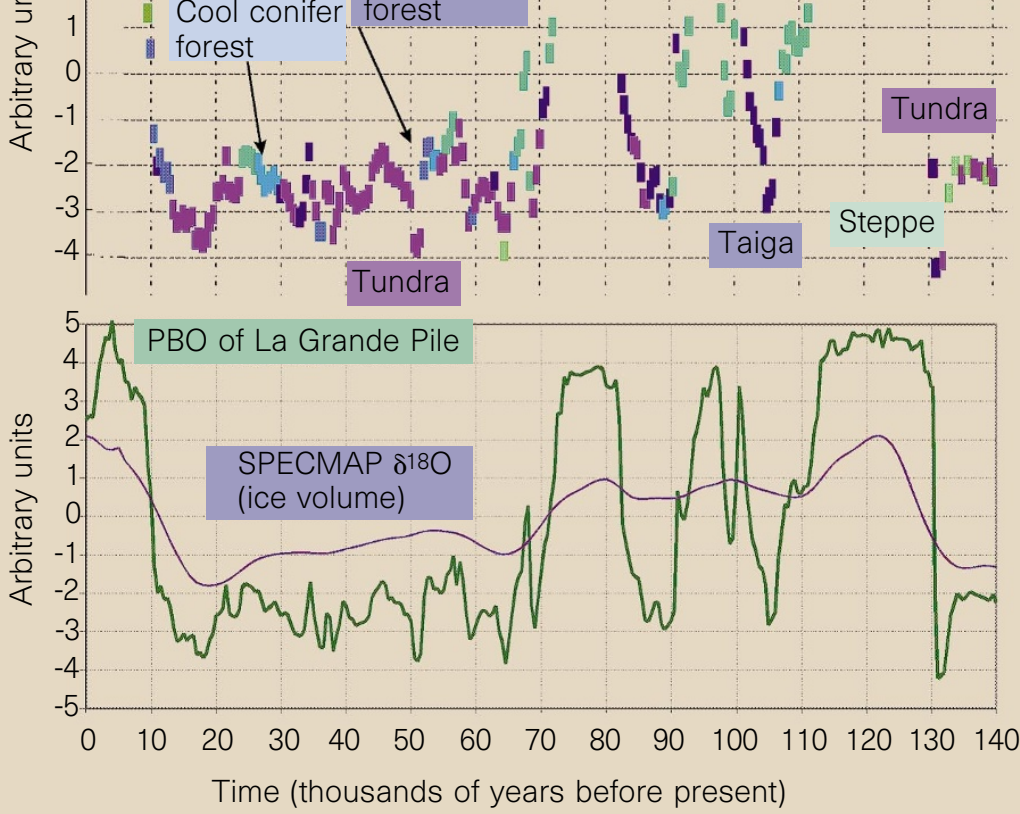

Figure 1 Succession of vegetation biomes in La Grande Pile, a pollen sequence from eastern France ${ }^{11}$, represented along a timescale ( $x$-axis), and the first principal component of pollen taxa (PBO) describing the main fluctuations (from forest to steppe/ tundra) in the pollen data. The biome succession was determined using the method of Prentice et al. ${ }^{13}$. The lower panel also shows ice volume over the same timescale, as represented by the same SPECMAP sequence used by Whitlock and Bartlein ${ }^{1}$ (see Fig. 2, page 58). 
and shrubs, due to a higher ice volume during a summer insolation maximum. As the authors point out, this last interglacialglacial cycle provides the possibility of examining the hierarchy of controls of past climate variations.

The notable point about the record analysed by Whitlock and Bartlein is its accurate dating. This was done with a good set of 13 dates from carbon-14 and volcanic-ash records. Moreover, although the pollen-zone boundaries have been assigned independently of the marine oxygen isotope stratigraphy (SPECMAP $\delta^{18} \mathrm{O}$, a proxy for global ice volume), their ages match the isotopic boundaries very nicely.

Accurate dating is, in fact, the crux of the matter, for lack of it has been the main handicap in analysis of the high-frequency fluctuations in vegetation history that are often inferred from continental (terrestrial) cores. Vegetation records are the products of interactions between physical, geological and biological systems, and the difficulty is in relating such records to a given climate mechanism. So it is not surprising that, in recent years, terrestrial palaeoecology has lagged behind palaeoceanography and glaciology, fields in which dating is less problematic and climate forcing can be interpreted more easily.

For example, most of the terrestrial cores contain a lot of high-frequency variations during the last glaciation, but the various explanations for such variations were rarely convincing. Only after glaciologists discovered the so-called Dansgaard-Oeschger events (temperate episodes in the glacial period), and palaeoceanographers discovered Heinrich events ${ }^{2}$ (colder events associated with iceberg discharges into the North Atlantic ocean), has it been possible to give a climatic explanation to the fluctuations in vegetation seen in continental cores. These climatic events have now been recognized in pollen and loess cores from Florida ${ }^{3}$ to China ${ }^{4}$. The message here is that we need first to understand the climate forcings before being able to interpret terrestrial data, and that these forcings cannot be discovered independently in the terrestrial cores.

Not least, interest in the abrupt climatic events of the past stems from how they might affect us in the future. The present interglacial period (the Holocene, which commenced only about 10,000 years ago) is being analysed to a finer and finer resolution, largely thanks to the wide availability of radiocarbon dates and the existence of annually laminated sediments. Clearly, however, the present interglacial is not yet finished; moreover, records of the course it has taken are sometimes disturbed by man. By contrast, the previous interglacial, stage $5 \mathrm{e}$, gives us the possibility of testing what happens as a glacial period begins by using climate model simulations ${ }^{5}$ and looking at long cores such as that from Carp Lake ${ }^{1}$. Such cores are rare, however, most of them coming from Europe ${ }^{6-9}$ or South America ${ }^{10}$ and often with a timescale dependent on the marine stratigraphy.

The main findings of Whitlock and Bartlein can be generalized to such cores, as illustrated in Fig. 1 by the pollen diagram of La Grande Pile in eastern France ${ }^{11}$. If we transform this pollen diagram into an index ${ }^{12}$ representing fluctuations between forest and herbaceous vegetation, or into a succession of biomes, the correlation with the global climate is evident for timescales higher than 20,000 years - even if, in this case, the timescale has not been derived independently of the ice-volume timescale. The difference between stages $5 \mathrm{e}$ and $5 \mathrm{a}$ that Whitlock and Bartlein point to can also be seen in this figure. The absence of deciduous forest in stage $5 \mathrm{a}$ is likely to be due to cooler winters, which themselves stem from higher ice volume and a cooler Atlantic Ocean.

We need more long sequences, especially in regions which are highly sensitive to climatic changes (for example, the monsoon regions), and analysis of them with sufficient

resolution to detect abrupt events compatible with a human timescale. The climate mechanisms revealed by these records can then be tested with climatic models, so as to help understand the plausibility of future abrupt changes and their effect on the environment.

Joël Guiot is in the CNRS UA 1152, Faculté de St-Jérôme, F-13397 Marseille cedex 20, France. e-mail:Joel.Guiot@lbhp.u-3mrs.fr

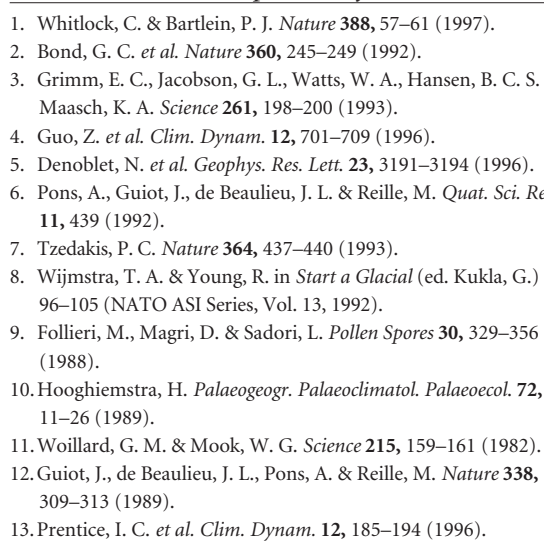

\section{Environmental engineering}

The case of the vanishing beaches
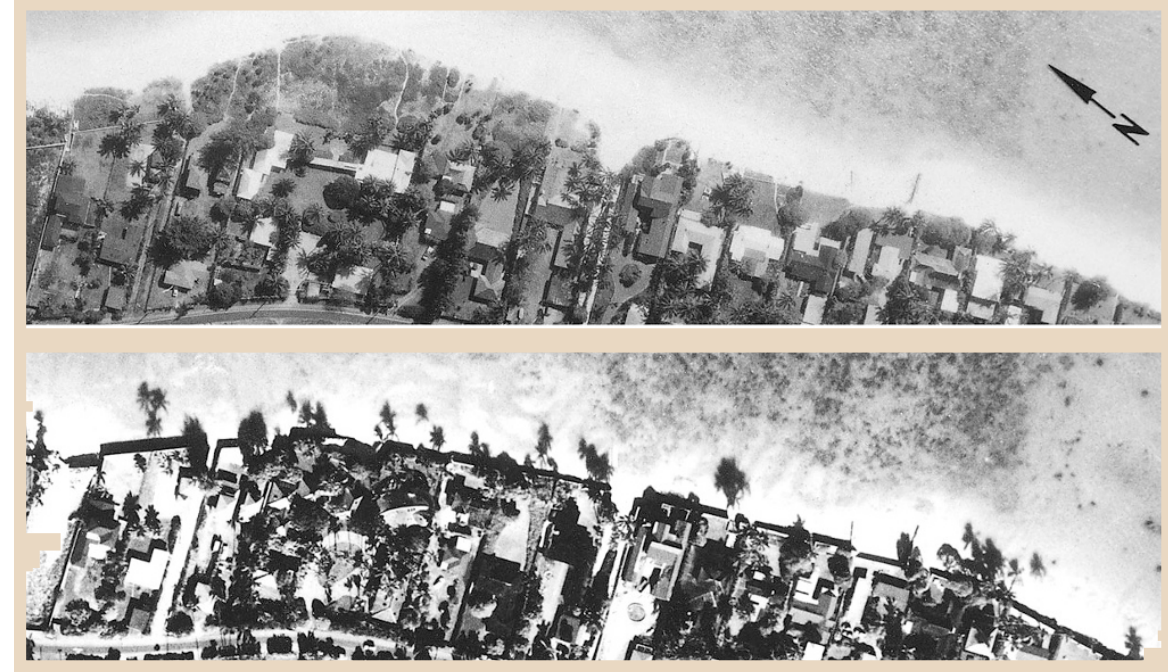

Now (top photograph, 1975) you see it; now (bottom, 1990) you don't. The 'it' is a beach, southeast of Lanikai on the Hawaiian island of Oahu, which disappeared over this period. This and other instances of beach loss or narrowing on Oahu are recorded by Fletcher, Mullane and Richmond in the Journal of Coastal Research (13, 209-215; 1997).

The authors identify coastal armouring structures, built to protect shoreline properties from erosion, as the culprits. The trouble is that armouring concentrates erosional forces on the beach directly in front, which, moreover, also loses the replenishment of sand stores from those locked into shoreline land. In all, the authors estimate that about 24 per cent of the 115 kilometres of Oahu's beaches have been lost or badly affected in this way over the mean (27-year) interval of their study.

Of the consequences discussed by the authors, one is transparent: the possible effects on the Hawaiian economy of a reduction in the number of tourists, who are largely drawn to the islands by the beaches. The 'visitor industry' exceeds by three times that of all others combined in terms of the revenue it delivers to the State of Hawaii.

There are no easy or cheap answers. And as Fletcher and colleagues point out, in a world in which rising sea levels may become an increasing threat, beach loss caused by structures designed to prevent coastal erosion is likely only to increase. Tim Lincoln 